\title{
Proportional Downscaling of Glutamatergic Release Sites by the General Anesthetic Propofol at Drosophila Motor Nerve Terminals
}

\author{
Shanker Karunanithi, ${ }^{1,2}$ Drew Cylinder, ${ }^{1}$ Deniz Ertekin, ${ }^{1}$ COressia H. Zalucki, ${ }^{3}$ Leo Marin, ${ }^{4}$ \\ Nickolas A. Lavidis, ${ }^{3}$ Harold L. Atwood, ${ }^{4}$ and ${ }^{-B}$ Bruno van Swinderen ${ }^{1}$
}

https://doi.org/10.1523/ENEURO.0422-19.2020

${ }^{1}$ Queensland Brain Institute, The University of Queensland, Brisbane 4072, Queensland, Australia, ${ }^{2}$ School of Medical Sciences, The University of New South Wales, Sydney 2052, New South Wales, Australia, ${ }^{3}$ School of Biomedical Sciences, The University of Queensland, Brisbane 4072, Queensland, Australia, and ${ }^{4}$ Department of Physiology, University of Toronto, Toronto, Ontario K1P 1J1, Canada

\section{Visual Abstract}
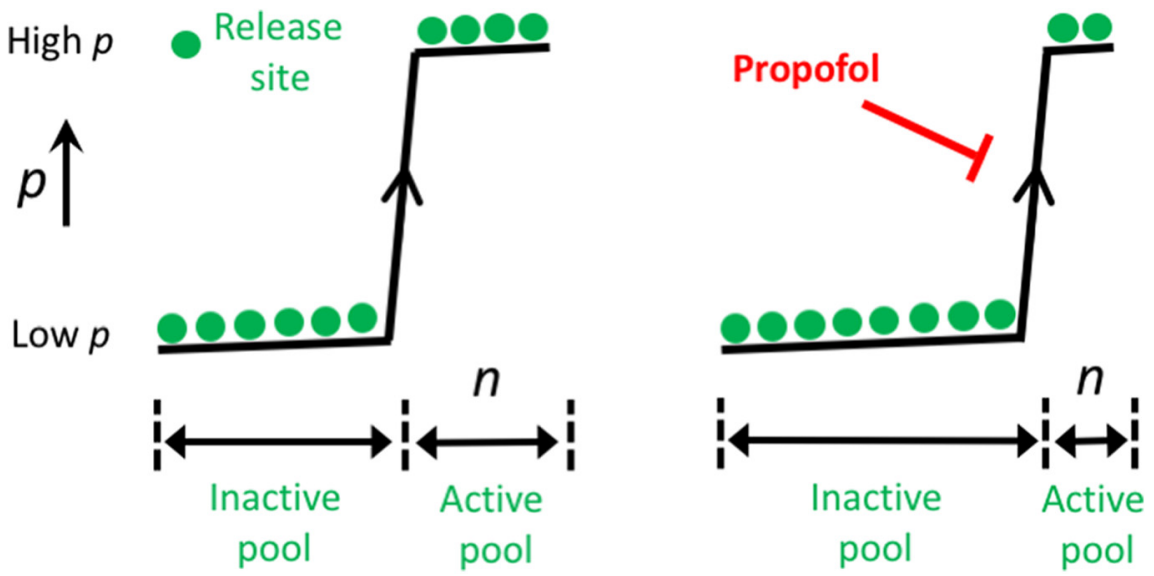

Propofol is the most common general anesthetic used for surgery in humans, yet its complete mechanism of action remains elusive. In addition to potentiating inhibitory synapses in the brain, propofol also impairs excitatory neurotransmission. We use electrophysiological recordings from individual glutamatergic boutons in male and female larval Drosophila melanogaster motor nerve terminals to characterize this effect. We recorded from two bouton types, which have distinct presynaptic physiology and different average numbers of release sites or active zones. We show that a clinically relevant dose of propofol $(3 \mu \mathrm{M})$ impairs neurotransmitter release similarly at both bouton types by decreasing the number of active release sites by half, without affecting release probability. In contrast, an analog of propofol has no effect on glutamate release. Coexpressing a truncated syntaxin1A protein in presynaptic boutons completely blocked this effect of propofol. Overexpressing wild-type syntaxin $1 \mathrm{~A}$ in boutons also conferred a level of resistance by increasing the number of active release sites to a physiological ceiling set by the number of active zones or T-bars, and in this way counteracting the effect of propofol. These results point to the presynaptic release machinery as a target for the general anesthetic. Proportionally equivalent effects of propofol on the number of active release sites across the different bouton types suggests that glutamatergic circuits that involve smaller boutons with fewer release sites may be more vulnerable to the presynaptic effects of the drug.

Key words: Drosophila melanogaster; electrophysiology; general anesthesia; neuromuscular junction; neurotransmission; syntaxin1A 


\section{Significance Statement}

Over 200 million surgeries are performed worldwide under general anesthesia every year, and the anesthetic of choice is increasingly the intravenous agent propofol. Sedation produced by propofol is understood to result from postsynaptic activation of inhibitory mechanisms in the brain. We have identified a presynaptic effect of propofol on excitatory synapses. Recording from individual glutamatergic boutons in fly larvae, we found that a clinically relevant dose of propofol impairs glutamate release by proportionally decreasing the participation of release sites across different bouton types. This suggests that propofol anesthesia involves presynaptic as well as postsynaptic mechanisms, and a proportional effect in large and small boutons may explain why some circuits are more vulnerable than others to the effects of the drug.

\section{Introduction}

In the 3 decades since its discovery, propofol has become the most commonly used general anesthetic for surgical procedures in humans. Like many other general anesthetics, propofol is understood to potentiate inhibitory pathways in the brain by acting on postsynaptic $\mathrm{GABA}_{\mathrm{A}}$ receptor subunits (Franks, 2008). Thus, an increasingly accepted idea for propofol-induced sedation is that it engages endogenous sleep pathways in the brain (Hemmings et al., 2019). However, sleep pathways cannot be the entire explanation, since-unlike sleep-surgical anesthesia is by definition not reversible as long as an adequate dose of the drug is applied. Additionally, there is growing evidence that general anesthesia more broadly is physiologically different from sleep, when investigated at the level of the neural circuitry involved (Brown et al., 2011) and the associated brain activity measures (Akeju and Brown, 2017). This suggests alternate target mechanisms that might complement the better understood processes relating to postsynaptic $\mathrm{GABA}_{\mathrm{A}}$ receptor function.

Recent work has shown that GABA-acting drugs such as propofol target a number of proteins other than $\mathrm{GABA}_{\mathrm{A}}$ receptors, including TrpA1 channels (Ton et al., 2017) and kinesin (Woll et al., 2018), resulting in parallel consequences

Received October 12, 2019; accepted January 24, 2020; First published February 4, 2020.

The authors declare no competing financial interests.

Author contributions: S.K. and B.v.S. designed research; S.K., D.C., D.E., O.H.Z., and B.v.S. performed research; L.M., N.A.L., and H.L.A. contributed unpublished reagents/analytic tools; S.K., D.C., D.E., O.H.Z., L.M., and B.v.S. analyzed data; S.K. and B.v.S. wrote the paper.

B.v.S. was supported by National Health and Medical Research Council Grants GNT1103923 and GNT1164879. S.K. was supported by Australian Research Council Grant LEl130100078 and grants from Griffith University.

Acknowledgments: We thank the members of the van Swinderen laboratory (Queensland Brain Institute) for feedback on the manuscript. We also thank Andrew Moorhouse (University of New South Wales) for discussion and help with analysis of mEJC kinetics. S.K. and B.v.S. thank Griffith University for the loan of the electrophysiology equipment used for this work.

Correspondence should be addressed to Bruno van Swinderen at b.vanswinderen@uq.edu.au.

https://doi.org/10.1523/ENEURO.0422-19.2020 Copyright (C) 2020 Karunanithi et al.

This is an open-access article distributed under the terms of the Creative Commons Attribution 4.0 International license, which permits unrestricted use, distribution and reproduction in any medium provided that the original work is properly attributed. on neural functions alongside sleep induction. Another emerging target mechanism is the presynaptic release machinery (van Swinderen and Kottler, 2014). There is increasing evidence in animal models as well as cell culture preparations that general anesthetics also act presynaptically (van Swinderen et al., 1999; Metz et al., 2007; Herring et al., 2009, 2011; Xie et al., 2013; Baumgart et al., 2015; Zalucki et al., 2015; Troup et al., 2019). Exactly how general anesthetics impair neurotransmission remains unclear, with isoflurane affecting presynaptic sodium and calcium channels (Koyanagi et al., 2019; Zhou et al., 2019) as well as SNARE formation (Xie et al., 2013; Troup et al., 2019); however, it is unknown whether intravenous and volatile agents might have common presynaptic effects. Work in rat neurosecretory PC12 cells showed that clinical concentrations of both propofol and isoflurane inhibit neurotransmission from these cells, and that coexpression of a mutant form of the presynaptic protein syntaxin1A preserves neurotransmission in these cells under anesthetic exposure (Herring et al., 2009, 2011). Syntaxin1A is a key membrane-bound component of the presynaptic release machinery, which is necessary to enable vesicular release of neurotransmitters (Südhof and Rizo, 2011). The effect of general anesthetics on syntaxin1A remained unclear, however, until the advent of super-resolution microscopy techniques that permitted visualizing and tracking single molecules through time (McKinney et al., 2009). In a recent super-resolution imaging study of a similar PC12 cell preparation, we confirmed a presynaptic effect of propofol and showed that the intravenous anesthetic impairs neurotransmission by restricting the mobility syntaxin $1 \mathrm{~A}$ on the cell membrane, resulting in nonfunctional nanoclusters (Bademosi et al., 2018). The same syntaxin1 A clustering effect was also found in a live animal preparation, namely motonerve terminals of the fly larval neuromuscular junction (NMJ; Bademosi et al., 2018), suggesting a common presynaptic target mechanism across different cell types and animal species. Together, these results also suggested an anesthetic effect more proximal to the presynaptic release machinery, at least for some intravenous agents such as propofol. One compelling hypothesis emerging from these findings is that propofol-impaired neurotransmitter release could result from decreased availability of syntaxin $1 \mathrm{~A}$ at release sites on the plasma membrane. Release sites, also referred to as "active zones," are where the 
presynaptic machinery comes together to facilitate the fusion of synaptic vesicles with the cell membrane (Atwood, 2006; Neher and Brose, 2018). Although multiple studies have now shown that general anesthetics impair neurotransmission, what remains unclear is how exocytosis physiology is affected in "real" synapses, in live animals. In the current study, we return to the fly larval NMJ to test how a clinically relevant dose $(3 \mu \mathrm{M})$ of propofol impairs glutamate release from individual motonerve terminals, or boutons. We further assess how coexpression of mutant and wild-type syntaxin1A protein modulate these presynaptic effects.

\section{Materials and Methods}

\section{Fly stocks}

Drosophila melanogaster strains were maintained on standard yeast-based medium at $22^{\circ}$ on a $12 \mathrm{~h}$ light/dark cycle. Flies were grown at low density to promote larval health. Food was kept moist with drops of additional distilled water as required, to promote burrowing in developing larvae. Strains used in this study were wild-type Canton S (CS), Elav-Gal4, UAS-syx-227 (Troup et al., 2019), UAS-syx-FL (Troup et al., 2019). Transgenic strains were outcrossed to a $\mathrm{w}^{1118}$ wild-type background. To generate syntaxin1A mutant or wild-type overexpression animals, homozygous UAS-syx-227 or UAS-syx-FL males were crossed to homozygous elavGal4 female virgins.

\section{Preparation of larvae for electrophysiological recordings}

Electrophysiological experiments were performed on muscle 6 , abdominal segment 3 , at room temperature $\left(22^{\circ} \mathrm{C}\right)$ in wandering third instar larvae of either sex. This muscle is innervated by $1 \mathrm{~b}$ and $1 \mathrm{~s}$ motoneurons, which are functionally different. Experiments were conducted in HL3 hemolymph-like physiological solution of the following composition (in mM): $70 \mathrm{NaCl}, 5 \mathrm{KCl}, 1 \mathrm{CaCl}_{2}, 20$ $\mathrm{MgCl}_{2}, 10 \mathrm{NaHCO}_{3}, 5$ trehalose, 115 sucrose, and $5 \mathrm{BES}$ [(N,N-bis(2-hydroxyethyl)-2-aminoethane sulfonic acid)] or HEPES, pH 7.2 and $\sim 340$ mOsm. The $1.5 \mathrm{~mm} \mathrm{CaCl}_{2}$ was used for some experiments. Karunanithi et al. (2018) provides detailed descriptions of the preparation of the larvae for electrophysiological recordings, the focal macropatch recording technique used for recording postsynaptic currents from single boutons, the assessment of the quality postsynaptic current recordings, the analysis and interpretation of the results, and the method for perfusing drugs into the recording chamber. Data from both sexes was combined.

\section{Focal macropatch recordings from single boutons: rationale}

The most explicit requirement for conducting proper quantal analysis is that the evoked and spontaneous postsynaptic responses be recorded from the synapses formed by a single input neuron (Del Castillo and Katz, 1954); and ideally at a single release site (Bekkers, 1994). Adhering to that requirement enables proper estimates of quantal parameters, namely quantal content (QC), quantal size, release probability, and active release site numbers, that being irrespective of the method used, assuming that that method adheres to requirements for proper quantal analysis (McLachlan, 1978; Korn and Faber, 1991; Clements, 2003). Meeting that requirement is not straightforward in larval muscles. This is because the larval muscles are multiply innervated (Hoang and Chiba, 2001). Virtually all larval muscles receive synaptic inputs from the highly active $1 \mathrm{~b}$ and the less active 1s motoneurons (Chouhan et al., 2010; Newman et al., 2017). The $1 \mathrm{~b}$ and $1 \mathrm{~s}$ motor nerve terminals are composed of boutons, which form synapses with the muscle (Atwood et al., 1993). The $1 \mathrm{~b}$ boutons form more synapses than $1 \mathrm{~s}$ boutons with the muscle (Atwood et al., 1993). Also, compared with 1s boutons, $1 \mathrm{~b}$ boutons exhibit smaller evoked and spontaneous mean postsynaptic response amplitudes, smaller release probabilities (Karunanithi et al., 2002; Pawlu et al., 2004; Dawson-Scully et al., 2007; Newman et al., 2017; Li et al., 2018), but higher active release site numbers (this article). Finally, $1 \mathrm{~b}$ boutons are facilitating, whereas 1s boutons are depressing (Kurdyak et al., 1994; Newman et al., 2017). Considering the significant difference between $1 \mathrm{~b}$ and $1 \mathrm{~s}$ synaptic properties, commonly used recording methods at the larval NMJ, namely, intracellular recordings and two-electrode voltage clamping (TEVC) cannot discriminate between the $1 \mathrm{~b}$ and 1s postsynaptic responses, and will yield inaccurate estimates of quantal parameters.

To circumvent such problems, we used the focal macropatch recording technique (FMRT) to unambiguously record postsynaptic currents from individual $1 \mathrm{~b}$ and $1 \mathrm{~s}$ boutons (Karunanithi et al., 2002, 2018; Pawlu et al., 2004; Kittel et al., 2006; Dawson-Scully et al., 2007). Like any technique, it needs to be performed correctly to get the proper results. In the case of FMRT, focal macropatch electrodes need to be fabricated in a manner to enable it to sit flush on the surface of the muscle fiber, without damaging it, when recording postsynaptic currents from the enclosed bouton (Karunanithi et al., 2018). The smaller evoked [excitatory junctional current (EJC)] and spontaneous [miniature EJC (mEJC)] mean amplitudes at $1 \mathrm{~b}$ boutons than at $1 \mathrm{~s}$ boutons were first observed using this technique (Karunanithi et al., 2002; Pawlu et al., 2004; Dawson-Scully et al., 2007) and were subsequently confirmed using optical quantal analysis (Newman et al., 2017; Li et al., 2018). Using FMRT, it was also shown that quantal amplitudes varied linearly with vesicle sizes, indicating that quantal amplitudes clearly reflected the transmitter content of individual synaptic vesicles and that postsynaptic receptors were unsaturated by a quantum of neurotransmitter (Karunanithi et al., 2002). Clear resolution of quantal amplitudes and of their variation at a selected synapse type is a prerequisite for quantal analysis (Korn and Faber, 1991), and those findings added confidence in using FMRT. The large signal-to-noise ratios observed in recordings of quantal currents indicate that no additional signal processing is required to remove contaminating noise from the signals during analysis, greatly simplifying analysis (Karunanithi et al., 2002, 2018). 
Mostly, a single vesicle is released from a single release site at low-frequency nerve stimulation (Melom et al., 2013), and the released neurotransmitter does not saturate postsynaptic receptors (Karunanithi et al., 2002). The evoked postsynaptic responses from single boutons are therefore not saturated responses, but proportional to the number of vesicles released from active release sites (Melom et al., 2013).

At larval NMJs, optical quantal analysis holds great promise to enable quantal analysis at not only singlebouton resolution, but also at single-release site resolution (Melom et al., 2013; Newman et al., 2017; Li et al., 2018). However, we chose not to use optical analysis because of the following drawbacks. (1) The responses are extremely protracted in time course, lasting hundreds of milliseconds and being 10-20 times the time course of quantal currents recorded using TEVC or FMRT (Bademosi et al., 2018; Li et al., 2018). Such responses are unphysiological since they do not reflect the speed of responses, which are characteristic of glutamatergic synapses. Such protracted time courses are also detrimental toward studying mechanisms of short-term presynaptic plasticity, which operate at submillisecondto-millisecond timescales (Regehr, 2012; Kaeser and Regehr, 2017). (2) Estimates of quantal parameters using optical analysis at single boutons could be inaccurate if inadequate numbers $(<10)$ of events are collected. Normally, adequate numbers of evoked (150-300 events) and spontaneous (25-50 events) postsynaptic responses are needed to correctly account for the variation in response amplitudes to conduct proper quantal analysis. Since the frequency of spontaneous events occurring at single larval NMJ boutons is very low, $\sim 0.04 \mathrm{~Hz}$ (Karunanithi et al., 1999; Bademosi et al., 2018), it would take $\sim 10 \mathrm{~min}$ to collect a minimum of 25 events. Continuous imaging for 10 min would likely bleach the preparation, resulting in misestimates of quantal parameters. (3) There has been no correlation made for optical imaging to establish that variations in quantal amplitude truly reflect the variations in released neurotransmitter from individual vesicles. In that regard, FMRT offers significant advantages for our analysis in making proper estimates of quantal parameters at the level of single synaptic boutons.

\section{Focal macropatch recordings from single boutons: methodology}

A brief account of the methods for conducting focal macropatch recordings are provided as follows. The preparation was viewed with a $60 \times$ water-immersion lens [numerical aperture (NA), 1.0] using Nomarski optics. Live images of the nerve terminals were captured using a lowlight video camera and projected onto a computer monitor. Such a setup enabled the selection of well isolated, single $1 \mathrm{~b}$ and $1 \mathrm{~s}$ boutons for focal macropatch recordings and the positioning of the focal macropatch electrode at the site of recording. The $1 \mathrm{~s}$ and $1 \mathrm{~b}$ boutons were identified under Nomarski optics (Kurdyak et al., 1994; Karunanithi et al., 2018). The $1 \mathrm{~b}$ nerve terminals are composed of larger and more variable-sized boutons (3-5 $\mu \mathrm{m}$ in diameter) than are $1 \mathrm{~s}$ nerve terminals (boutons, $\sim 3 \mu \mathrm{m}$ in diameter; Atwood et al., 1993; Kurdyak et al., 1994). Well isolated $1 \mathrm{~s}$ or $1 \mathrm{~b}$ boutons were selected for recordings. The focal macropatch electrodes possessed open tip diameters of approximately $\sim 5 \mu \mathrm{m}$ and were filled with HL3 solution. The open tip does not exert direct pressure onto the bouton during recordings (Karunanithi et al., 2002). The recorded signals were amplified using an Axoclamp 900A amplifier (under bridge/l-clamp mode; Molecular Devices), digitized (at an acquisition rate of 40 $\mathrm{kHz}$ ), and stored on a computer using the PowerLab 4/35 data acquisition system (ADInstruments). In each experiment, focal macropatch recordings were made from a single $1 \mathrm{~b}$ or a single $1 \mathrm{~s}$ bouton, or from two boutons of each type. In each experiment, 200 EJCs and 25$30 \mathrm{mEJCs}$ were recorded from a single bouton. The stimulating electrode (lumen diameter, $\sim 10-12 \mu \mathrm{m}$ ), filled with perfusate, was used to gently suck the cut end of the segmental nerve and was stimulated at a frequency of $1 \mathrm{~Hz}$ at supramaximal strengths to evoke EJCs.

The measured EJC and mEJC amplitudes were used to derive quantal size ( $q$; average $\mathrm{mEJC}$ amplitude), average EJC amplitude, QC (average EJC amplitude/average mEJC amplitude), and the quanta released per stimulus. In each experiment, the quanta released per stimulus were calculated by dividing each EJC amplitude in the train by the average $\mathrm{mEJC}$ amplitude (Bademosi et al., 2018). The cumulative relative frequency histogram for each group represents the pooled data from all the experiments in that group. For each experiment, QC should equal the average quanta released per stimulus.

At high $\left[\mathrm{Ca}^{2+}\right]_{0}$, neurotransmitter release can be described by a binomial model, where QC $=n p$ (Del Castillo and Katz, 1954; McLachlan, 1978). $n$ equates to the average number of functionally active release units/sites, and $p$ equates to the average release probability of those active sites (Lavidis, 1995). In each experiment, $p$ was calculated using the following equation:

$$
p=1-\frac{S^{2}}{Q C q^{2}}+\frac{\sigma^{2}}{q^{2}} .
$$

where $q$ is the quantal size, $S^{2}$ is the variance of the EJC amplitudes, and $\sigma^{2}$ is the variance of the mEJC amplitudes (Bennett and Florin, 1974; McLachlan, 1978; Lavidis and Bennett, 1992). $n$ was calculated by dividing QC by $p$.

For measuring quantal release kinetics (mEJC rise times and decay constants), we used LabChart Pro software (version 8.1.5; ADInstruments). The mEJC decay time constant was measured by fitting a single exponential to the mEJC decay, using the Peak Analysis subroutine. Fits of decay were manually checked for accuracy. Rise times were measured manually, using the two cursors placed at the base and the peak of each mEJC, as done previously (Karunanithi et al., 1999).

\section{Drug perfusion}

A syringe pump (KDS 100 Legacy Syringe Pump, KD Scientific) was used to perfuse the preparation with perfusate throughout all experiments (Zalucki et al., 2015). Perfusion was conducted at rates of 1-2 $\mathrm{ml} / \mathrm{min}$ over 30 
$40 \mathrm{~min}$ before recordings were performed and also throughout the recording session. In each experiment, recordings were brief, taking $\sim 3.5 \mathrm{~min}$ for EJCs and 5-10 min for mEJCS. When drugs were to be perfused into the bath, stock concentrations of propofol and the propofol analog 2,4-diisopropylphenol (Sigma-Aldrich) were prepared in DMSO at $0.4 \% \mathrm{v} / \mathrm{v}$ concentration and then dissolved into the HL3 solution. To ensure that perfusate had equilibrated in the animal tissue, preparations were exposed to propofol-containing perfusate (or analog-containing solutions) before recording electrode placement. Similarly, in control experiments, the preparations were perfused with the perfusate (lacking propofol) before recording electrode placement. The focal macropatch electrode was also left immersed in the perfusate before the recording session, ensuring that the drug concentration within the lumen of the electrode equilibrated to that outside of it. By taking such measures and by considering that the period of recording was brief relative to the period of perfusion, the drug concentration within the lumen of the electrode could be considered as being stable. Similar procedures were undertaken in control experiments; however, the perfusate lacked drugs. Drug concentrations were in a clinically relevant range $(3 \mu \mathrm{M})$, as determined previously (Bademosi et al., 2018). Higher propofol concentrations (30 $\mu \mathrm{m})$ led to a $50 \%$ failure rate per stimulus in this preparation (compared with $0 \%$ failure rate for $3 \mu \mathrm{M}$ ), suggesting that at supraclinical drug concentrations, mechanisms other than just neurotransmitter release were also being compromised, such as failures of nerve action potentials to invade the motor nerve terminals and the attenuation of nerve-evoked calcium influx. We therefore restricted our quantal analysis in this study to $3 \mu \mathrm{M}$ propofol, which was unaffected by action potential failures.

\section{Serial electron microscopy used to determine T-bars per bouton}

Experiments and analyses were performed as previously described (Karunanithi et al., 2002) to determine the number of T-bars/active zones per bouton on muscle 6 , segment A3 in CS larvae. Three larvae were serially sectioned and used for analysis. The thickness of each section was $75 \mathrm{~nm}$. A series of thin sections were cut along muscle 6 , and measurements of T-bar numbers per bouton made from $1 \mathrm{~b}$ and $1 \mathrm{~s}$ boutons within those series. Electron micrographs along a series were used to serially reconstruct the string of boutons, along with some of their key structural features. A bouton was identified as a structure whose cross-sectional area changed approximately fivefold at the beginning and at the end from its midcross-sectional area (Lnenicka et al., 1986). Within each bouton, synapses were identified as those electron-dense presynaptic and postsynaptic membranes in close apposition, and T-bars were those dense projections located on the presynaptic membrane, surrounded by a cloud of synaptic vesicles. We determined the number of T-bars per bouton by counting the number of T-bars located on synapses within each bouton.

\section{Immunochemistry}

Wandering third instar larvae were dissected in fresh extracellular fluid and fixed for 20 min with cold $4 \%$ paraformaldehyde in $1 \times$ PBS. The preparation was blocked with $0.2 \%$ Triton $\mathrm{X}-100,2 \%$ bovine serum albumin (Jackson ImmunoResearch), or $10 \%$ normal goat serum (SigmaAldrich) in PBS-T (1× PBS, 0.2 Triton X-100) for $30 \mathrm{~min}$. The following antibodies were used: HRP-Cy3 (1:120; Jackson ImmunoResearch), Bruchpilot (mouse anti-nc82, 1:100; Developmental Studies Hybridoma Bank), and HA (rabbit anti-HA, 1:1000; Cell Signaling Technology). Primary antibody incubations were performed at room temperature for $1 \mathrm{~h}$. Secondary antibodies were diluted in blocking buffer in PBS-T [Invitrogen Alexa Fluor 488 goat anti-mouse (1:250) and Alexa Fluor 647 goat anti-rabbit, Thermo Fisher Scientific]. Samples were incubated with secondary antibodies for $3 \mathrm{~h}$. The preparations were mounted in Vectashield (Vector Laboratories). Imaging of the nerve terminals innervating muscle 6 was performed on a Zeiss 710 Meta confocal microscope and on a spinning-disk confocal system (Marianas, 3l) consisting of an Axio Observer Z1 (Carl Zeiss) equipped with a CSU-W1 spinning-disk head (Yokogawa Corporation of America), ORCA-Flash4.0 version 2 sCMOS camera (Hamamatsu Photonics) using a $63 \times 1.4$ NA PlanApo oil-immersion objective. Mouse HA-monoclonal antibody $(1: 3000)$ was used to tag HA-Syntaxin1A and HA-Syntaxin1 $\mathrm{A}^{227}$. The secondary antibody was donkey anti-mouse Invitrogen Alexa Fluor 647 (1:500; Thermo Fisher Scientific).

\section{Quantification of active zone number}

Images were processed on Fiji (ImageJ) software. Approximately 20 is and $1 \mathrm{~b}$ boutons were selected per animal for quantification, 10 per side. Two blinded experimenters counted the number of puncta at the selected terminals independently. Representative images were deconvolved by using Huygens Pro software (Scientific Volume Imaging).

\section{Experimental design and statistical analysis}

Statistical and data analysis was performed using Excel (Microsoft) and Prism 6 (GraphPad Software) software. To assess statistical differences between two groups, the unpaired (two-tailed) Student's $t$ test was used, with Welch's correction. The nonparametric tests, one-way ANOVA and the Kolmogorov-Smirnov (K-S) test were used to assess statistical differences. In all cases, significance was set at $p<0.05$. The $\mathrm{K}-\mathrm{S}$ test was used to compare the shapes of standardized distributions. Distributions were standardized by subtracting the average and then normalizing to $1 \mathrm{SD}$. In rank-ordered plots, values were rank ordered in ascending order and then plotted against one another (Turrigiano et al., 1998). The coefficient of variation was obtained by dividing the SD of a population by its average. Where possible, normalized parameters were provided in the figures to improve readability, with the non-normalized data provided as Extended Data. Parameters were normalized to their respective averages in controls. All data are available in the main text, figures, or the Extended Data. Raw data, 
A

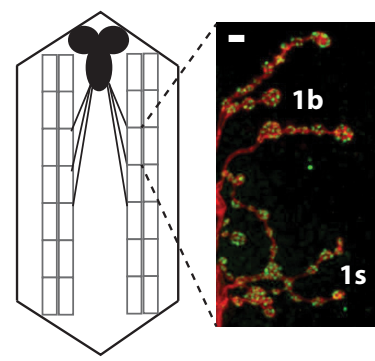

D
B
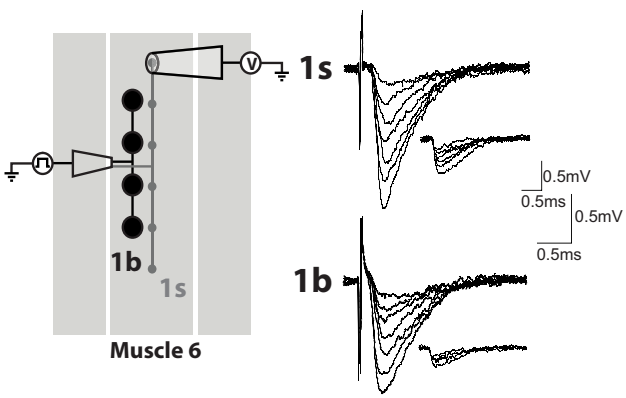

E
C

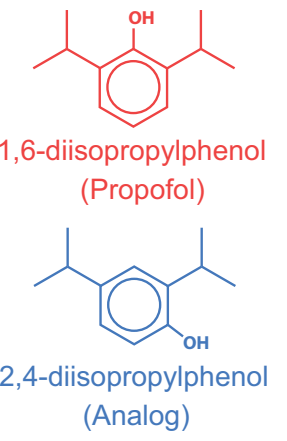

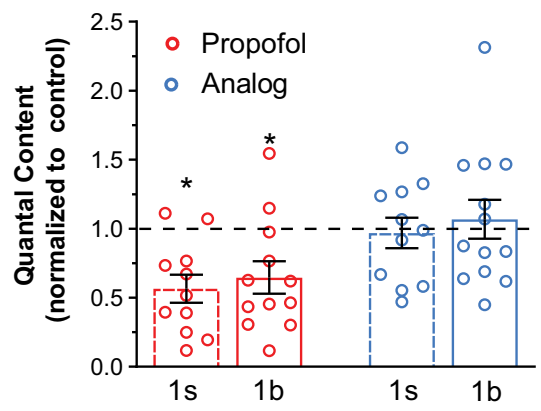

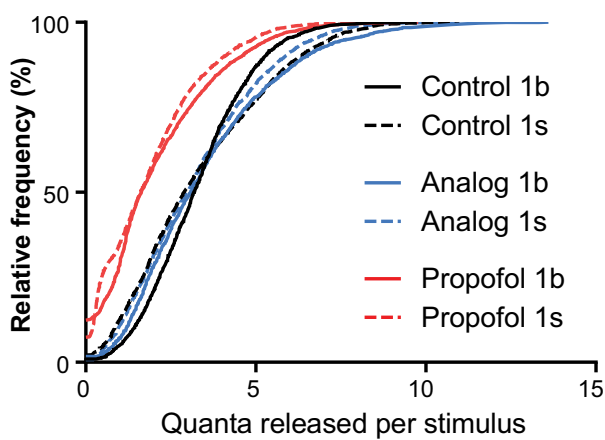

Figure 1. Propofol decreases glutamate release similarly from different synapse types. $\boldsymbol{A}$, Left, Schema of Drosophila larval NMJ preparation. The black structure is the brain, rectangles are muscle segments. Middle, Muscle 6 motor nerve terminals labeled with HRP to identify neurons and nc82 to identify active zones. 1s and $1 \mathrm{~b}$ boutons are indicated. Scale bar, $4 \mu \mathrm{m}$. Right, Diagram illustrating the configuration for focal macropatch recording from single $1 \mathrm{~s}$ and $1 \mathrm{~b}$ boutons at the fly larval NMJ. $\boldsymbol{B}$, Example traces of EJCs and mEJCs (inset) recorded from 1s and $1 \mathrm{~b}$ boutons in the CS strain. $\boldsymbol{C}$, Chemical structures of propofol (1,6-diisopropylphe-

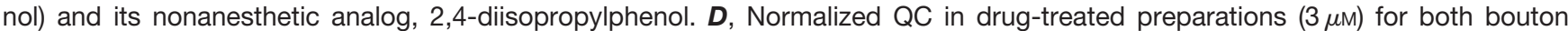
types. Parameters were normalized to their respective averages in non-drug-treated controls (dashed line). Raw data are presented in Extended Data Figure 1-1. $* p<0.05$; Student's $t$ test. Graphs show individual data points (animals), and error bars show the average \pm SEM. $\boldsymbol{E}$, Cumulative plot of quanta released per stimulus, for both bouton types and both drugs.

code, and materials used in the analysis are available on request from the corresponding author.

\section{Results}

\section{Propofol decreases glutamate release similarly at different bouton types}

To measure propofol effects on neurotransmitter release, we used electrophysiological recordings from individual glutamatergic boutons in Drosophila larvae (Karunanithi et al., 2018; Fig. 1A). The Drosophila larval NMJ is a highly studied model glutamatergic synapse that shares similarities with mammalian glutamatergic synapses (Menon et al., 2013; Harris and Littleton, 2015). Fly larval NMJs are unusual in that virtually all the muscles receive inputs from two distinct motoneurons (Hoang and Chiba, 2001), as follows: the larger, highly active $1 \mathrm{~b}$ and the smaller, less active 1s motoneurons (Chouhan et al., 2010; Newman et al., 2017; Fig. 1A). The nerve terminals of $1 \mathrm{~s}$ and $1 \mathrm{~b}$ motoneurons are composed of synaptic boutons, which form synapses with the muscle (Atwood et al., 1993; Newman et al., 2017). Nearly all nerve terminals on the presynaptic side possess release sites (or active zones) where neurotransmitter release occurs, and $1 \mathrm{~b}$ boutons possess on average more release sites than 1s boutons (Atwood et al., 1993; Kittel et al., 2006; Wagh et al., 2006). Despite this, $1 \mathrm{~b}$ boutons have lower release probabilities and generate smaller postsynaptic responses than 1s boutons (Kurdyak et al., 1994; Newman et al., 2017). The 1s and 1b synapses are therefore referred to as "strong" and "weak," respectively (Kurdyak et al., 1994; Atwood and Karunanithi, 2002; Newman et al., 2017).

We distinguished between the effects of propofol on $1 \mathrm{~b}$ and 1s neurotransmitter release by recording postsynaptic currents from single $1 \mathrm{~b}$ and $1 \mathrm{~s}$ boutons, using focal macropatch electrodes (Karunanithi et al., 2018; Fig. 1A; for details on why this recording preparation is appropriate for the questions posed here, see Materials and Methods). We recorded the stimulus-evoked EJCs and the mEJCs that resulted when individual vesicles spontaneously released neurotransmitter (Fig. 1B). From measures of the mean amplitudes of EJCs (estimating synaptic strength) and mEJCs (estimating quantal size) in each experiment, we determined the amount of neurotransmitter released by deriving QC (average number of vesicles released per stimulus; Karunanithi et al., 2018). These results confirmed that propofol (Fig. 1C) decreased QC at both bouton types (Fig. 1D,E and Extended Data 
A

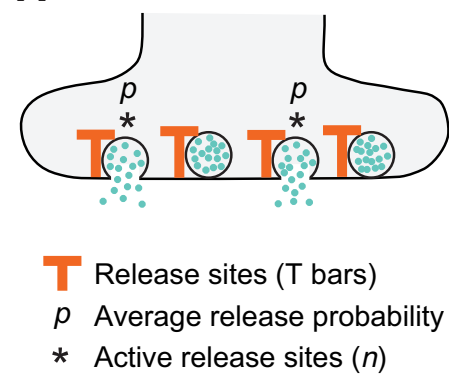

D

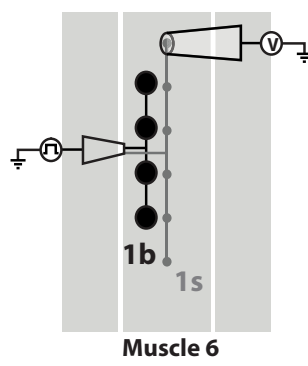

G
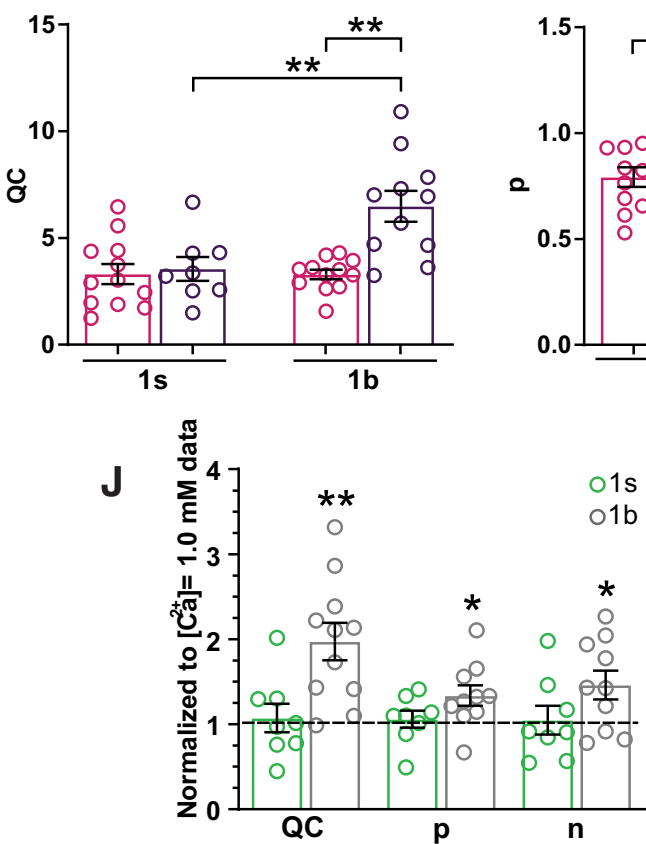

H
B

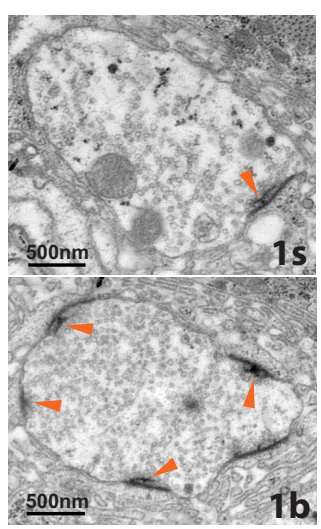

E

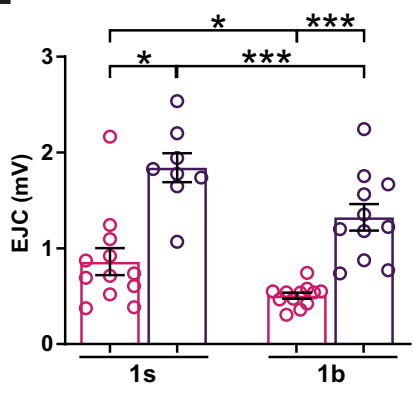

C

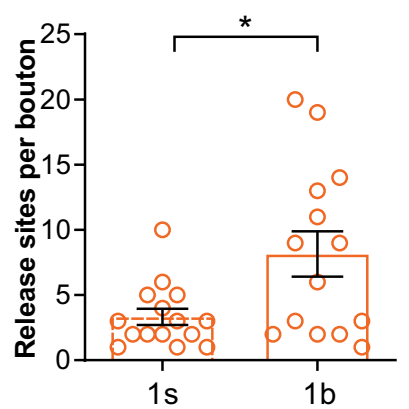

$01.5 \mathrm{mM} \mathrm{Ca}{ }^{2+}$

$01.0 \mathrm{mM} \mathrm{Ca}^{2+}$

F

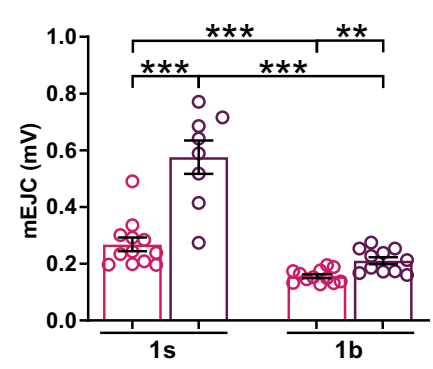

I
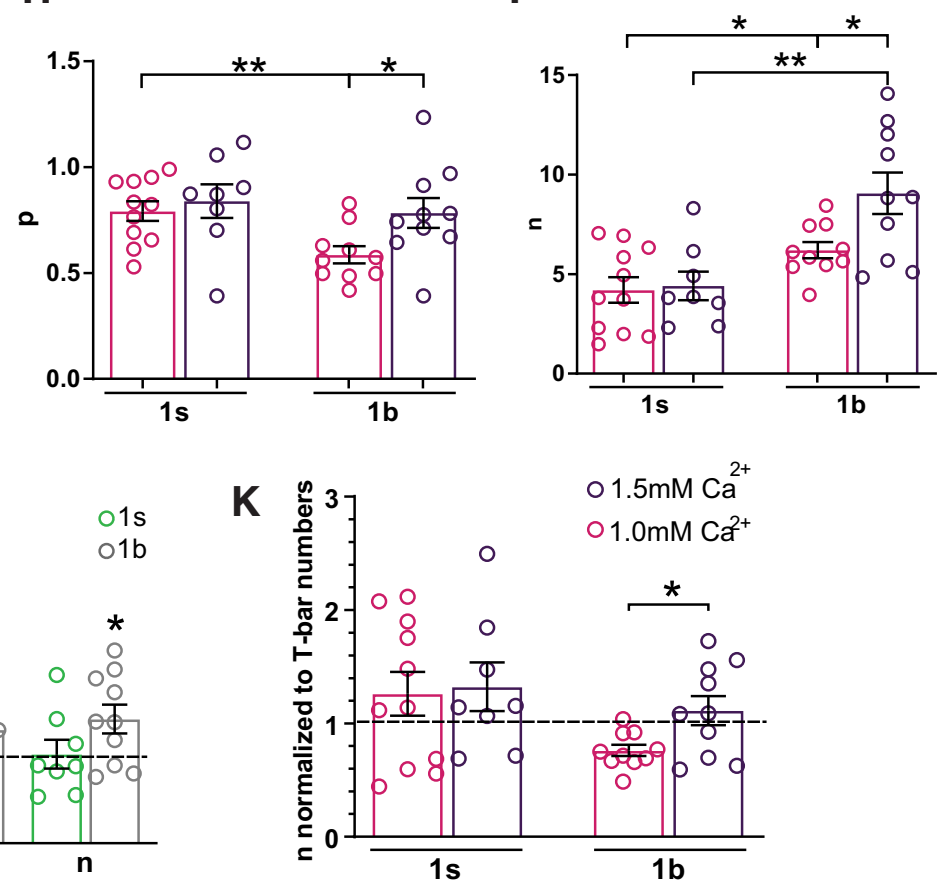

Figure 2. The upper limit for $n$ is set by the number of T-bars. $\boldsymbol{A}$, Schema of release sites at a synapse. Active release sites (*) contribute to the average release p. B. Electron micrographs (EMs) of a $1 \mathrm{~s}$ (top) and a $1 \mathrm{~b}$ (bottom) bouton, with T-bars (synapses) indicated with arrowheads. $\boldsymbol{C}$, Average number ( \pm SEM) of release sites for $1 \mathrm{~s}$ and $1 \mathrm{~b}$ boutons, determined from the T-bar counts from the EM data. See Materials and Methods, and Extended Data Figure 2-1 for other examples. $\boldsymbol{D}$, $\left[\mathrm{Ca}^{2+}\right]_{\mathrm{e}}$ was raised from 1.0 to 1.5 $\mathrm{mm}$ in focal macropatch recordings in CS larvae. Right, Sample recordings from single $1 \mathrm{~s}$ and $1 \mathrm{~b}$ boutons revealed increases in the amplitudes of EJCs and mEJCs at both bouton types under higher calcium concentrations. $\boldsymbol{E}$, Average EJC ( \pm SEM) for $1 \mathrm{~s}$ and $1 \mathrm{~b}$ recordings. $\boldsymbol{F}$, Average $\mathrm{mEJC}( \pm \mathrm{SEM})$ for $1 \mathrm{~s}$ and $1 \mathrm{~b}$ recordings. $\boldsymbol{G}$, As $\left[\mathrm{Ca}^{2+}\right]_{\mathrm{e}}$ was raised, calculations of $\mathrm{QCs}$ revealed no increase in 1s QC, whereas there was an increase in $1 \mathrm{~b}$ QC. $\boldsymbol{H}, \boldsymbol{I}$, There were no increases in $p(H)$ or in $n(I)$ at $1 \mathrm{~s}$ boutons, whereas there 
continued

were increases in both $p(\boldsymbol{H})$ and $n(\boldsymbol{I})$ at $1 \mathrm{~b}$ boutons, contributing to the increase in $1 \mathrm{~b}$ QC $(\boldsymbol{G})$. $\boldsymbol{J}$, We observed that at $1 \mathrm{~s}$ boutons, all the release sites were already activated at $1.0 \mathrm{~mm}\left[\mathrm{Ca}^{2+}\right]_{\mathrm{e}}$, with no additional increase being observed when $\left[\mathrm{Ca}^{2+}\right]_{\mathrm{e}}$ was further raised. At $1 \mathrm{~b}$ boutons, not all of the release sites were activated at $1.0 \mathrm{~mm}\left[\mathrm{Ca}^{2+}\right]_{\mathrm{e}}$, whereas they were all activated when $\left[\mathrm{Ca}^{2+}\right]_{\mathrm{e}}$ was further raised. $\boldsymbol{K}$, We generated a graph to test whether values of $n$ approached the number of T-bars per bouton as $\left[\mathrm{Ca}^{2+}\right]_{\mathrm{e}}$ was raised, by normalizing $n$ (Fig. $2 l$ ) to the number of T-bars per bouton (Fig. $2 C$ ). The dashed line at 1.0 is representative of when $n$ equates to the number of T-bars per bouton. These results indicate that $n$ is limited by the number of T-bars per bouton type. $* p<0.05 ; * * p<0.01 ; * * * p<0.001$; Student's $t$ test. $1.0 \mathrm{~mm}\left[\mathrm{Ca}^{2+}\right]_{\mathrm{e}}$ data are the same as in Figure 1, shown here for comparison. Graphs show individual data points (animals), and all error bars are \pm SEM.

Fig. 1-1A-C; Bademosi et al., 2018). In contrast, an analog of propofol, 2, 4-diisopropylphenol (Fig. 1C), had no effects on QC (Fig. 1D,E and Extended Data Fig. 1-1A-C). A more detailed examination of the number of quanta released per stimulus showed a consistent decrease under propofol but no change for the analog, compared with controls (Fig. 1E). In conclusion, glutamate release appears similarly impaired by propofol at $1 b$ and 1 s synapses, while the analog does not appear to affect glutamatergic neurotransmission in this preparation.

We further tested whether propofol affected mEJC frequency and postsynaptic receptor kinetics. $1 \mathrm{~b}$ and $1 \mathrm{~s}$ mEJC frequencies were unaffected by either propofol or its analog (Extended Data Fig. 1-1D), indicating that propofol did not affect spontaneous vesicle fusion. At mammalian inhibitory synapses, propofol prolongs the decay of inhibitory postsynaptic currents (Kitamura et al., 2003). To determine whether propofol was acting directly on postsynaptic current kinetics, we analyzed mEJC rise and decay times (Extended Data Fig. 1-1E). Propofol did not affect either the averaged rise times or the decay time constants at either bouton type (Extended Data Fig. 1-1F,G). The analog did, however, modestly prolong both those parameters at $1 \mathrm{~b}$ boutons (Extended Data Fig. 1-1F,G).

\section{Determining the number of active release sites in $1 \mathrm{~s}$ and $1 \mathrm{~b}$ boutons}

We next investigated how propofol decreased QC at individual boutons. This first required estimating two key aspects of QC, namely the number of active release sites per bouton, and their associated release probability. At high $\left[\mathrm{Ca}^{2+}\right]_{\mathrm{e}}(1 \mathrm{~mm})$, which is close to physiological concentrations (Atwood and Karunanithi, 2002), QC is described by the equation $Q C=n p$, where $n$ represents the average number of functionally active release sites, and $p$ represents the average release probability (average chance of a vesicle releasing neurotransmitter at an active release site, following a stimulus; Del Castillo and Katz, 1954; McLachlan, 1978; see Materials and Methods). Stimulation at low frequencies, as used here, causes neurotransmitter to be released from a single vesicle at a single release site (Melom et al., 2013). The model of quantal release from a bouton can thus be visualized as vesicles releasing neurotransmitter from a population of $n$ active release sites with an average probability, $p$ (McLachlan, 1978; Fig. 2A). Within boutons, the anatomic correlates of release sites are the T-bars (Kittel et al., 2006; Wagh et al., 2006; Fig. 2B and Extended Data Fig. 2-1). We found that the upper limit for the number of active release sites per bouton was set by the number of T-bars, which were approximately four in 1s boutons and eight in $1 \mathrm{~b}$ boutons, although there was considerable variability within bouton types (Fig. 2B,C and Extended Data Fig. 2-1). To ascertain that our physiological estimates of $n$ conformed with the actual physical number of active zones per bouton, we recorded from $1 \mathrm{~s}$ and $1 \mathrm{~b}$ boutons under increased calcium concentrations $(1.5 \mathrm{~mm})$ to maximize neurotransmission (Fig. 2D), and calculated $n$ and $p$ for these data, compared with physiological $(1.0 \mathrm{~mm})$ data. Increasing the calcium concentration increased EJC and mEJC amplitudes in both $1 \mathrm{~s}$ and $1 \mathrm{~b}$ recordings (Fig. $2 E, F$ ), although quantal content was only increased in $1 \mathrm{~b}$ (Fig. 2G). Release probability $(p)$ and the number of active release sites $(n)$ remained unchanged in $1 \mathrm{~s}$, whereas both increased in $1 \mathrm{~b}$ (Fig. $2 H, I)$. Increasing calcium thus had no effect on 1s boutons, but significantly increased the number of active release sites in 1b, and therefore quantal content (Fig. 2J). Importantly, raising calcium to $1.5 \mathrm{~mm}$ increased $n$ to the physical ceiling set by the average number of T-bars expected in $1 \mathrm{~b}$ boutons (approximately eight), whereas $n$ remained at the ceiling already achieved in 1s boutons (approximately four) at physiological calcium concentrations (Fig. 2K). This shows that, at calcium concentrations of $1.0 \mathrm{~mm}$, all the release sites are active in 1s boutons, whereas only a fraction are active in $1 \mathrm{~b}$ boutons, but when the calcium concentration was raised further to $1.5 \mathrm{~mm}$, all the release sites in both $1 \mathrm{~s}$ and $1 \mathrm{~b}$ boutons became active. This also shows that our physiological calculations of $n$ match well with the actual anatomic number of release sites observed in either bouton type. Our accurate assessment of $n$ allowed us to then evaluate the effect of propofol on glutamate release at physiological $\left[\mathrm{Ca}^{2+}\right]_{\mathrm{e}}$ : is propofol decreasing $\mathrm{QC}$ by decreasing release $p$, the $n$ of active release sites, or both?

\section{Propofol decreases the number of active release sites in $1 \mathrm{~s}$ and $1 \mathrm{~b}$ boutons}

Interestingly, propofol had the exact same effect at 1s and 1b boutons: it lowered QC by decreasing $n$ and not $p$ at both bouton types, whereas the analog, as expected, had no effects on these parameters (Fig. $3 A, B$ ). Normalizing the data to controls revealed that propofol had a proportionally equivalent effect on $1 \mathrm{~b}$ and $1 \mathrm{~s}$ boutons (Fig. $3 C$ ), decreasing the number of functionally active release sites $(n)$ by half, without affecting the release probability $(p)$. The fact that $p$ did not change under propofol exposure indicates that the remaining active release sites were not compromised. These uncompromised release sites probably contributed to the large variation in 
A

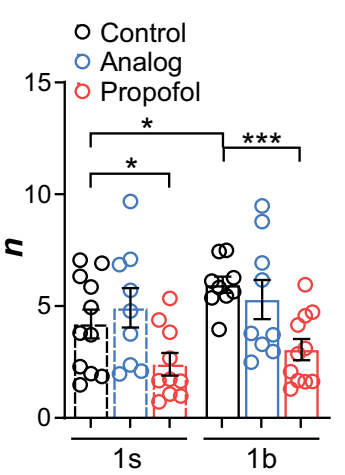

D

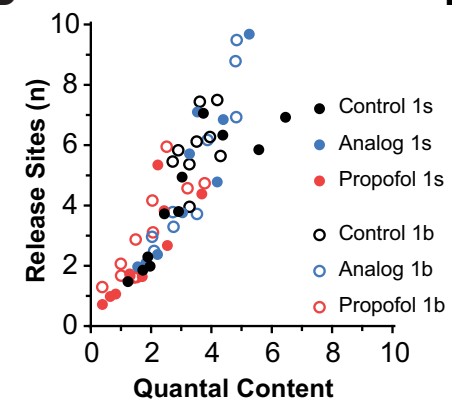

B

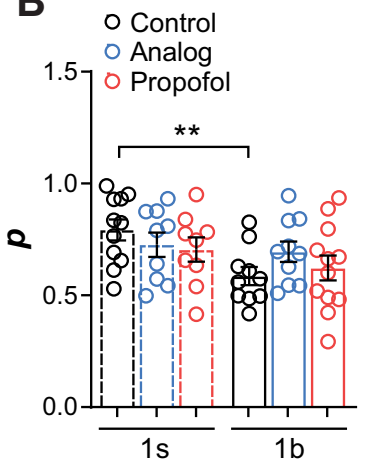

E

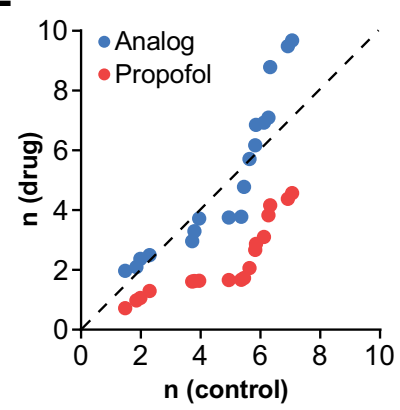

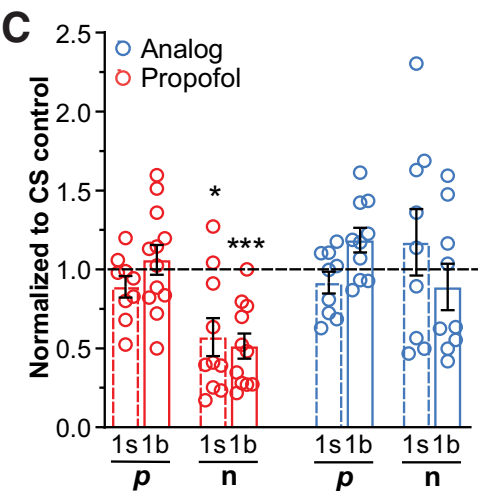

$\mathbf{F}$

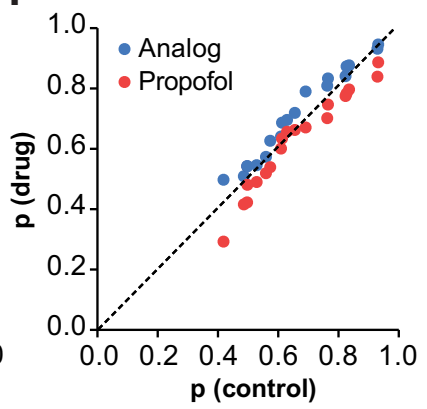

Figure 3. Propofol decreases the number of active release sites at synapses. $\boldsymbol{A}$, Estimated number of active release sites ( $n$; see Materials and Methods) in 1s and $1 \mathrm{~b}$ boutons, for control and drug-treated conditions, in CS animals. B, Estimated release probability ( $\boldsymbol{D}$, see "Materials and Methods") in 1s and 1b boutons, for control and drug-treated conditions, in CS animals. $\boldsymbol{C}$, Data from $\boldsymbol{A}$ and $\boldsymbol{B}$, normalized to non-drug-treated controls (dashed line). Graphs in $\boldsymbol{A}-\boldsymbol{C}$ are averages \pm SEM; individual data points (animals) are shown. $* p<0.05 ; * * p<0.01$; $* * * p<0.05$, Student's $t$ test. $\boldsymbol{D}$, Quantal content plotted against $n$, from all $1 \mathrm{~s}$ and $1 \mathrm{~b}$ recordings in control and drug conditions. $\boldsymbol{E}$, Rank-ordered plot of $n$ for drug versus control conditions. Dashed line indicates no effect of drug on $n$. $\boldsymbol{F}$, Rank-ordered plot of $p$ for drug versus control conditions. Dashed line indicates no effect of drug on $p$.

QC observed among preparations (Fig. 1D). If more release sites were compromised by propofol, the variations in QC would have been smaller, since neurotransmitter release would have been muted at some boutons.

We next plotted the relationship between $n$ and QC across all of our experiments (Fig. $3 D$ ) to better understand how $n$ was distributed among our data. Although $1 \mathrm{~s}$ and $1 \mathrm{~b}$ boutons have on average a different number of active zones (Fig. 2C and Extended Data Fig. 2-1), we observed a linear relationship between $n$ and QC across all tested conditions when data from $1 \mathrm{~s}$ and $1 \mathrm{~b}$ boutons were combined (Fig. 3D). This showed considerable overlap between bouton types, suggesting that knowing the baseline $n$ is more important for detecting the effect of the drug, rather than knowing what the bouton type was. In other words, the number of active release sites is highly variable per bouton, and the difference between 1s and $1 \mathrm{~b}$ boutons is hardly discrete in this regard. We therefore next examined whether boutons possessing specific numbers of functionally active release sites were more affected than others by propofol, irrespective of bouton type. To do this, we rank ordered (in ascending order) $n$ derived from control and from propofol-treated preparations and plotted them against one another (Turrigiano et al., 1998; Fig. 3E). Points clustered close to the dashed line represent cases where there were no or minimal effects of propofol. Consistent with our previous analysis
(Fig. 3C), we found that propofol decreased the number of active release sites by about half for most values of $n$, with the strongest effects observed in boutons displaying an $n$ value between 4 and 6 (Fig. 3E). As anticipated, we did not observe any such effects with the analog (Fig. 3E). As neither propofol nor its analog had an effect on release probability, we observed all points clustered on the dashed line in rank-ordered plots for $p$ (Fig. 3F). These results indicate that the effect of propofol on neurotransmission is linked to the number of functionally active release sites available, irrespective of bouton type.

\section{Syntaxin1A manipulations confer resistance to propofol by preserving or increasing the number of active release sites}

Release sites require a variety of molecular infrastructure, including the target SNARE syntaxin1A (Südhof and Rizo, 2011), and components of the synaptic release machinery have been implicated in modulating general anesthesia phenotypes (Xie et al., 2013; van Swinderen and Kottler, 2014; Bademosi et al., 2018). Experiments in cultured neurosecretory cells have shown that the effects exerted by propofol on neurotransmitter release can be completely blocked by coexpressing a truncated syntaxin $1 \mathrm{~A}$ protein alongside endogenous syntaxin1A (Herring et al., 2011; Bademosi et al., 2018). Similar truncated syntaxin1A proteins have also 
A
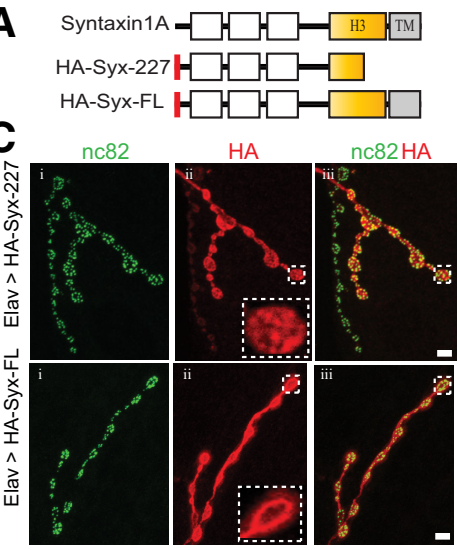

$\mathbf{F}$

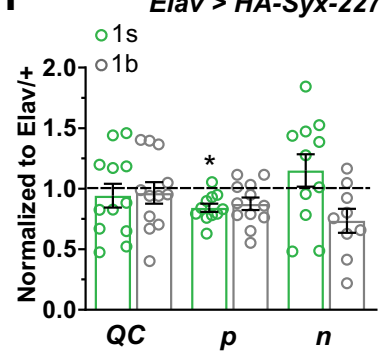

Elav > HA-Syx-FL

I

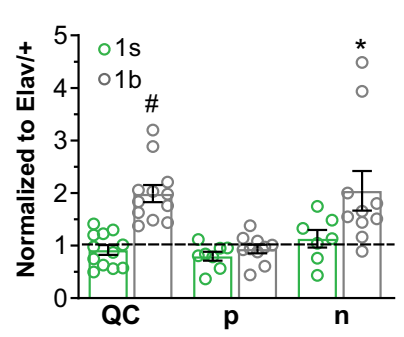

L

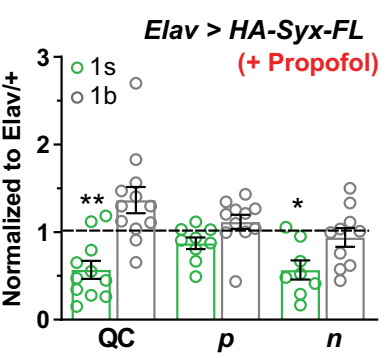

B

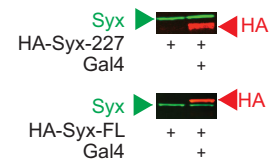

D $\circ$ Elav/ +

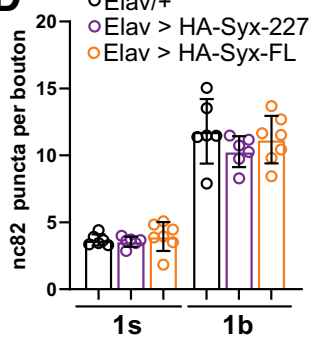

E

E $1 \mathrm{~s}$

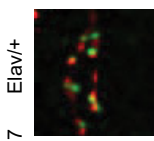

$1 b$
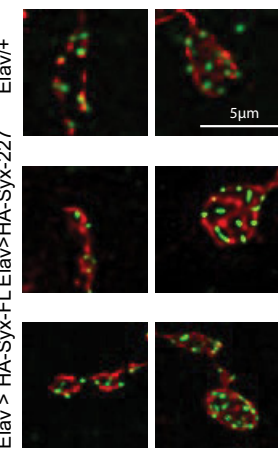

nc82 HRP
G

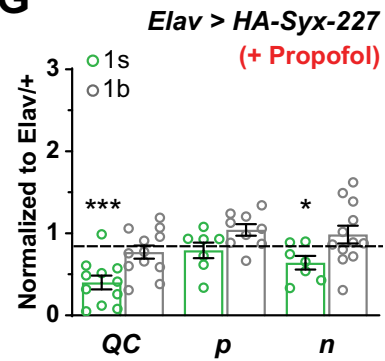

H
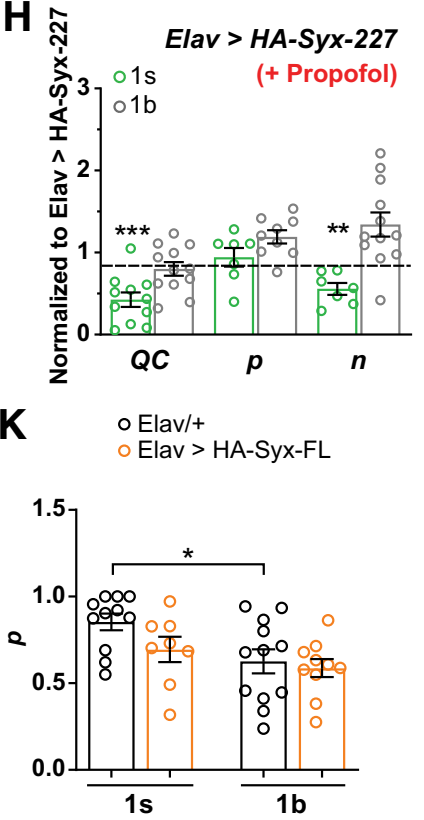

N

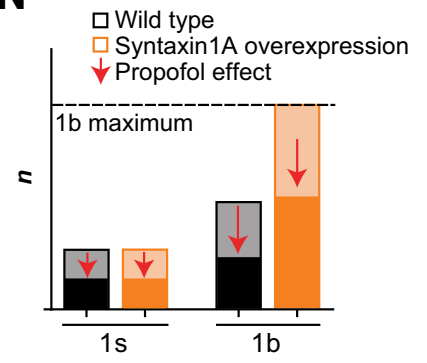

Figure 4. Syntaxin1A manipulations confer resistance to propofol by preserving or increasing the number of active release sites. $\boldsymbol{A}$, Schematic representing the wild-type syntaxin1A protein, an HA-tagged truncated syntaxin1A construct (HA-Syx-227), and an HAtagged full-length syntaxin1A construct (HA-Syx-FL). H3, SNARE interaction domain; TM, transmembrane domain. $\boldsymbol{B}$, Coexpression of the truncated and full-length constructs in Drosophila shown by Western blot: endogenous wild-type syntaxin1A (green), HA-Syx227, and HA-Syx-FL (red). C, Immunolabeling of larval boutons in HA-Syx-227 and HA-Syx-FL: left column, synaptic marker, nc82 (green); middle column, HA-labeling of coexpressed syntaxin1A (red, magnification of a single bouton is inset, showing localization of the coexpressed truncated syntaxin1A protein); right column, merge of nc82 and HA labels. Scale bar, $4 \mu \mathrm{m}$. $\boldsymbol{D}$, Average number of active zones $( \pm$ SEM) in Elav $>H A-S y x-227(N=6)$ and Elav $>H A-S y x-F L$ animals $(N=7)$ compared with Elav/+ controls $(N=6)$, for $1 \mathrm{~s}$ and $1 \mathrm{~b}$ boutons. $\boldsymbol{E}$, Example boutons (labeled with HRP) and puncta (labeled with nc82) for the same strains as in $\boldsymbol{D}$. $\boldsymbol{F}, \mathrm{QC}, \boldsymbol{p}$ and $n$ for the two bouton types in Elav > HA-Syx-227, normalized to Elav/+ (dashed line; Extended Data Fig. 4-1). G, QC, $p$ and $n$ for the two bouton types in Elav > HA-Syx-227 + propofol, normalized to Elav/+ (dashed line; Extended Data Fig. 4-2). $\boldsymbol{H}, \mathrm{QC}, \boldsymbol{p}$ and $n$ for the two bouton types in Elav > HA-Syx-227 + propofol, normalized to HA-Syx-227 without propofol (dashed line; Extended Data Fig. 4-3). I, QC, $p$ and $n$ ( \pm SEM) for the two bouton types in Elav $>H A-S y x-F L$, normalized to Elav/+ (dashed line; Extended Data Fig. 4-4). J, Average number ( \pm SEM) of estimated active release sites $(n)$ in Elav $>H A-S y x-F L$ animals, for $1 \mathrm{~b}$ and 
continued

1s boutons. $\boldsymbol{K}$, Average estimated release probability ( $\boldsymbol{D} \pm \mathrm{SEM}$ ) in Elav $>H A-S y x-F L$ animals, for $1 \mathrm{~b}$ and $1 \mathrm{~s}$ boutons. $\boldsymbol{L}$, QC, $p$ and $n$ ( \pm SEM) for the two bouton types in Elav > HA-Syx-FL + propofol, normalized to Elav/+ (dashed line; Extended Data Fig. 4-5). $M, Q C, p$ and $n$ ( \pm SEM) for the two bouton types in Elav $>H A-S y x-F L+$ propofol, normalized to $H A-S y x-F L$ without propofol (dashed line; Extended Data Fig. 4-6). Individual data points in $\boldsymbol{F}-\boldsymbol{M}$ are animals. $* p<0.05 ; * * p<0.01 ; * * * p<0.001, \# p<0.0001$, Student's $t$ test. $\boldsymbol{N}$, Summary schema of the opposing effects of syntaxin1A overexpression and propofol on the number of active release sites $(n)$. Physiologic maxima for $1 \mathrm{~b}$ boutons is indicated. Darker shading below arrows represents the proportional decrease in $n$, under propofol exposure.

been shown to block the effect of volatile anesthetics in vivo as well as in vitro (van Swinderen et al., 1999; Metz et al., 2007; Herring et al., 2009). We therefore investigated whether the coexpression of a truncated syntaxin1A protein in Drosophila larvae could block the specific effect of propofol that we have uncovered here, as this would confirm that preserving $n$ is key to conferring resistance to the presynaptic effects of the drug. To test this, we expressed a similarly truncated syntaxin1A in Drosophila (HA-Syx227; Fig. 4A), driven by the UAS/Gal4 system (Duffy, 2002). We inserted an $\mathrm{HA}$ tag onto the $\mathrm{N}$ terminus of the protein (Fig. 4A, red bar) to enable us to verify its expression in the wild-type syntaxin1A background. We confirmed that the truncated syntaxin1A protein is expressed in Drosophila larvae (Fig. 4B, top) and then examined its localization in motoneurons by colabeling with the active-zone marker bruchpilot (nc82; Wagh et al., 2006; Fig. 4C). Interestingly, although HA-Syx-227 was strongly expressed in $1 \mathrm{~b}$ nerve terminals, expression levels in 1s boutons were low to nondetectable (Fig. 4C, top row). This differential expression pattern gave us an opportunity to compare the effects of propofol on manipulated versus "control" nerve terminals within the same animals. Importantly, coexpressing the truncated syntaxin1A protein did not change the average number of anatomic release sites (active zones) per bouton, compared with controls (Fig. 4D,E).

In the absence of propofol, coexpression of truncated syntaxin1A revealed no significant change in baseline $\mathrm{QC}$ or $n$ compared with control animals (Elav $>$ Syx-227 normalized to Elav/+), for $1 \mathrm{~b}$ or $1 \mathrm{~s}$ boutons (Fig. $4 F$ and Extended Data Fig. 4-1A-E). We did note, however, a decrease in ECJ and mEJC amplitudes in 1s boutons, as well as a decrease in $p$ (Extended Data Fig. 4-1A, $B, E$ ), which suggests a residual transgenic effect in these smaller boutons. In the presence of propofol, QC and $n$ were decreased only at the 1s boutons, whereas $Q C$ and $n$ remained at control levels in the $1 b$ boutons that strongly expressed the truncated syntaxin1A protein (Fig. $4 G$ and Extended Data Fig. 4-2A-E). We next assessed propofol effects within the same Elav $>S y x-227$ strain. Consistent with previous studies in cell culture (Herring et al., 2011; Bademosi et al., 2018), coexpression of the truncated syntaxin1A construct completely blocked the effect of propofol on neurotransmitter release from $1 \mathrm{~b}$ boutons expressing the mutant protein (Fig. $4 H$ and Extended Data Fig. 4-3A-E), whereas in control 1s boutons (in the same animals) the number of active release sites was decreased by half (Fig. 4H).

If propofol decreases the number of active release sites, then increasing this number should reset the effect of the drug and thereby confer a level of resistance. We therefore sought a way to artificially increase the number of active release sites in nerve terminals. We hypothesized that providing synapses with a surplus of wild-type syntaxin1A might increase the number of active release sites per bouton. To test this, we expressed a full-length (FL) wild-type syntaxin1A in Drosophila (HA-Syx-FL; Fig. $4 A)$. We again inserted an $\mathrm{HA}$ tag onto the $\mathrm{N}$ terminus of the protein (Fig. $4 A$, red bar) to enable us to track its expression in an endogenous syntaxin1A background. We verified that the transgenic wild-type syntaxin1A was indeed expressed in Drosophila larvae (Fig. 4B, bottom), and then observed that the wild-type construct was expressed in both synaptic bouton types in motor nerve terminals (Fig. 4C, bottom row). Interestingly, overexpressing wild-type syntaxin1A did not increase the average number of active zones in $1 \mathrm{~s}$ or $1 \mathrm{~b}$ boutons, as measured by counting nc82 puncta in Elav $>H A-S y x-F L$ animals, compared with Elav/+ controls; the syntaxin1A overexpressing line exhibited $\sim 4$ and 10 puncta per $1 \mathrm{~s}$ and $1 \mathrm{~b}$ boutons, respectively (Fig. 4D,E).

We next assessed the effect of syntaxin1A overexpression on synaptic physiology in the absence of propofol, in Elav $>H A-S y x-F L$ animals compared with Elav/+ controls. We found that overexpression of syntaxin1A caused QC to be increased at $1 \mathrm{~b}$ but not at $1 \mathrm{~s}$ boutons (Fig. $4 /$ and Extended Data Fig. 4-4A-C). The increase in 1b QC resulted from an increase in $n$ and not in $p$ (Fig. 4I-K). $n$ was increased to $\sim 10$ in $1 \mathrm{~b}$ boutons (Fig. 4J), matching the average number of active zones in these boutons (Figs. $2 C$, $4 D)$. In $1 \mathrm{~s}$ boutons, $n$ remained the same, at approximately four per bouton (Fig. 4J), matching the average number of active zones in those boutons (Figs. 2C, 4D).

Syntaxin1A overexpression therefore recruits additional active release sites at $1 \mathrm{~b}$ boutons, but not $1 \mathrm{~s}$ boutons. Following our hypothesis, this genetic manipulation should reset the effect of propofol in $1 \mathrm{~b}$ boutons specifically. This is indeed what we observed: as $1 \mathrm{~b}$ boutons in syntaxin1 $\mathrm{A}-$ overexpressing (Elav $<H A-S y x-F L)$ animals had an increased $n$ value (Fig. 4J), the net effect of propofol in this strain was to return $n$ (and thus QC) to control (Elav/+) levels (Fig. $4 L$, dashed line), whereas the decrease in $n$ (and QC) at $1 \mathrm{~s}$ boutons was below control levels (Fig. $4 L$ and Extended Data Fig. 4-5A-E).

Despite this differential effect of syntaxin1A overexpression on $1 \mathrm{~b}$ and $1 \mathrm{~s}$ boutons, we found that propofol still decreased QC at both bouton types by similar proportions, in animals overexpressing syntaxin1A (Fig. $4 M$ and Extended Data Fig. 4-6A-C). This decrease in QC was due to equivalent effects on $n$ at both types of synapse, with no effect on $p$ (Fig. $4 M$ and Extended Data Fig. 4-6D, $E)$. Increasing the number of active release sites in $1 \mathrm{~b}$ 
boutons therefore does not change the net effect of propofol, which is to decrease $n$ by about half. Rather, $1 \mathrm{~b}$ boutons are relatively less impacted by the drug in Elav $<H A-S y x-F L$ animals because of an artificially higher $n$ baseline (Fig. 4J). In conclusion, propofol has a proportionally equivalent effect impairing neurotransmitter release from either type of synapse, but a capacity to increase the number of active release sites in $1 \mathrm{~b}$ boutons (Figs. 2l, 4J) counteracts its effect at these synapses specifically (Fig. $4 N$ ).

\section{Discussion}

In this work, we have provided evidence that a clinically relevant concentration of the intravenous general anesthetic propofol impairs excitatory neurotransmission by decreasing (by half) the availability of active release sites at presynaptic terminals. Our findings are intriguing because they point to a likely mechanism whereby drugs such as propofol might be impairing presynaptic release: not by decreasing the release probability of already primed (i.e., active) release sites, but instead by altering the ratio between active and inactive sites. Most presynaptic terminals involve a dynamic balance of active and inactive release sites, and this balance can vary across synapse types (Neher and Brose, 2018). By recording from individual boutons at the Drosophila NMJ, we have shown that knowing the $n$ value of a bouton is key to understanding the effect of propofol on neurotransmission. We show that even within similar bouton types $n$ can vary widely, so to capture the effect of propofol on $n$ requires a bouton-by-bouton assessment of the number of active release sites that are available. This would have been missed by simply averaging all bouton recordings or by only measuring combined $1 \mathrm{~s}$ and $1 \mathrm{~b}$ potentials recorded from postsynaptic tissue. Similarly, previous studies investigating presynaptic effects of propofol at the frog NMJ (Leite et al., 2011) or in rat cortical cultures (Kitamura et al., 2003) did not acquire physiological data allowing estimates of release site numbers, as we have done here. It is interesting to note, however, that propofol exposure increases quantal size in the both the fly (Extended Data Fig. 1-1B) and frog (Leite et al., 2011) NMJ.

Earlier work on the vertebrate NMJ showed that opioids and adenosine also inhibit neurotransmission by decreasing $n$ (Bennett et al., 1991; Lavidis, 1995). These compounds activate presynaptic autoreceptors, which then initiate a cascade of events leading to decreased neurotransmitter release via an estimated decrease in $n$. As in those earlier vertebrate studies, our estimates of $n$ in fly larval boutons are purely electrophysiological. However, our electrophysiological estimates of $n$ maxima match well with anatomic counts of T-bar numbers or BRP puncta in fly larval boutons, providing confidence that our estimates of the effect of propofol on $n$ are accurate. There are currently no reliable imaging or immunochemical techniques for disambiguating active from inactive release sites, although methods aimed at tracking the molecular dynamics and conformation of key vesicular priming proteins such as munc13 (Dittman, 2019) provide a promising future research direction to verify our electrophysiological observations.

One interpretation of our results is that propofol converts a proportion of active sites with a high release probability to inactive sites with low release probability. Alternatively, propofol could inhibit inactive sites from converting to active sites. Our data do not reveal the directionality of this effect, only that propofol increases the number of inactive sites without affecting the release probability of remaining active sites. Thus, release sites unaffected by propofol presumably remain fully functional (rather than half-functional, for example), as evidenced by our finding that release probability at the fly NMJ is not impaired by propofol. In previous studies, we have suggested that propofol may act on components of the synaptic release machinery prior to SNARE formation (Bademosi et al., 2018), which is consistent with our current finding that $n$ is affected rather than $p$. Additionally, we have shown that the resistance-inducing truncated syntaxin1A protein is not incorporated into the SNARE complex (Troup et al., 2019), which again suggests a role prior to SNARE formation. One possibility that we have raised before (Bademosi et al., 2018) is that propofol inhibits a step in the formation of functional release sites, thereby creating "traffic jams" of inactive sites that fail to become fully functional. A level of resistance to propofol is thus afforded at some boutons, depending on the availability of additional inactive release sites, which could be recruited to become active, depending on the physiological demands of a neuron. Thus, overexpressing syntaxin $1 \mathrm{~A}$ in $1 \mathrm{~b}$ boutons maintains glutamate release near wild-type levels in the presence of propofol because these larger boutons have additional release sites that can be recruited to become high-release probability (thus active) sites. On the other hand, coexpressing a truncated syntaxin $1 \mathrm{~A}$ in $1 \mathrm{~b}$ boutons preserves $n$ and thus QC from the effects of propofol specifically in these boutons, while $n$ is again halved in unprotected 1s boutons in the same tissue. Together, these genetic manipulations provide compelling evidence that, alongside its known effects on postsynaptic receptors and ion channels (Franks, 2008; Hemmings et al., 2019), propofol impairs neurotransmission by decreasing the availability of active release sites. Genetic manipulations of different components of the SNARE assembly and priming processes should clarify whether propofol renders active sites inactive, or whether it prevents inactive sites from becoming active.

Although synaptic release mechanisms are extremely conserved across animals (Südhof and Rizo, 2011), not all presynapses are the same. As we have shown here, even synapses releasing the same neurotransmitter (glutamate) onto the same muscle can be physiologically different. 1s boutons have a higher release probability (Figs. $2 \mathrm{H}, 3 \mathrm{~B}$ ), and all of their release sites are active (Fig. $2 \mathrm{~J}, K$ ), whereas $1 \mathrm{~b}$ boutons are weaker (Extended Data Fig. 1-1A) but have a capacity to recruit more release sites (Figs. 2/, 4J). Yet, both types of boutons are impacted in a similar way by propofol, with the drug decreasing the number of active release sites by half. If propofol impairs neurotransmitter release by proportionally decreasing $n$ by half, as we show here, then smaller boutons (e.g., 1s in our study) 
could be more vulnerable to the presynaptic effects of the drug, and this would especially be the case for synapses with only one active zone, which probably describes most excitatory synapses in the brain (Schikorski and Stevens, 1997). Extrapolating to humans, this may explain why some circuits are more affected than others by surgical concentrations of general anesthetics such as propofol (Kim et al., 2007). Future work should reveal whether the presynaptic effect that we have uncovered for propofol extends to other types of synapses (e.g., inhibitory) and neurotransmitter systems, and whether this effect may be a common presynaptic mechanism of other general anesthetics.

\section{References}

Akeju O, Brown EN (2017) Neural oscillations demonstrate that general anesthesia and sedative states are neurophysiologically distinct from sleep. Curr Opin Neurobiol 44:178-185.

Atwood HL, Govind CK, Wu CF (1993) Differential ultrastructure of synaptic terminals on ventral longitudinal abdominal muscles in Drosophila larvae. J Neurobiol 24:1008-1024.

Atwood HL (2006) Neuroscience. Gatekeeper at the synapse. Science 312:1008-1009.

Atwood HL, Karunanithi S (2002) Diversification of synaptic strength: Presynaptic elements. Nat Rev Neurosci 3:497-516.

Bademosi AT, Steeves J, Karunanithi S, Zalucki OH, Gormal RS, Liu $S$, Lauwers E, Verstreken $P$, Anggono V, Meunier FA, van Swinderen B (2018) Trapping of Syntaxin1a in presynaptic nanoclusters by a clinically relevant general anesthetic. Cell Rep 22:427-440.

Baumgart JP, Zhou ZY, Hara M, Cook DC, Hoppa MB, Ryan TA, Hemmings HC Jr (2015) Isoflurane inhibits synaptic vesicle exocytosis through reduced $\mathrm{Ca} 2+$ influx, not $\mathrm{Ca} 2+$-exocytosis coupling. Proc Natl Acad Sci USA 112:11959-11964.

Bekkers JM (1994) Quantal analysis of synaptic transmission in the central nervous system. Curr Opin Neurobiol 4:360-365.

Bennett MR, Florin T (1974) A statistical analysis of the release of acetylcholine at newly formed synapses in striated muscle. J Physiol 238:93-107.

Bennett MR, Karunanithi S, Lavidis NA (1991) Probabilistic secretion of quanta from nerve terminals in toad (Bufo marinus) muscle modulated by adenosine. J Physiol 433:421-434.

Brown EN, Purdon PL, Van Dort CJ (2011) General anesthesia and altered states of arousal: A systems neuroscience analysis. Annu Rev Neurosci 34:601-628.

Chouhan AK, Zhang J, Zinsmaier KE, Macleod GT (2010) Presynaptic mitochondria in functionally different motor neurons exhibit similar affinities for $\mathrm{Ca}^{2+}$ but exert little influence as $\mathrm{Ca}^{2+}$ buffers at nerve firing rates in situ. J Neurosci 30:1869-1881.

Clements JD (2003) Variance-mean analysis: A simple and reliable approach for investigating synaptic transmission and modulation. J Neurosci Methods 130:115-125.

Dawson-Scully K, Lin Y, Imad M, Zhang J, Marin L, Horne JA, Meinertzhagen IA, Karunanithi S, Zinsmaier KE, Atwood HL (2007) Morphological and functional effects of altered cysteine string protein at the Drosophila larval neuromuscular junction. Synapse 61:1-16.

Del Castillo J, Katz B (1954) Quantal components of the end-plate potential. J Physiol 124:560-573.

Dittman JS (2019) Unc13: A multifunctional synaptic marvel. Curr Opin Neurobiol 57:17-25.

Duffy JB (2002) GAL4 system in Drosophila: A fly geneticist's Swiss army knife. Genesis 34:1-15.

Franks NP (2008) General anaesthesia: From molecular targets to neuronal pathways of sleep and arousal. Nat Rev Neurosci 9:370386.
Harris KP, Littleton JT (2015) Transmission, development, and plasticity of synapses. Genetics 201:345-375.

Hemmings HC Jr, Riegelhaupt PM, Kelz MB, Solt K, Eckenhoff RG, Orser BA, Goldstein PA (2019) Towards a comprehensive understanding of anesthetic mechanisms of action: A decade of discovery. Trends Pharmacol Sci 40:464-481.

Herring BE, Xie Z, Marks J, Fox AP (2009) Isoflurane inhibits the neurotransmitter release machinery. J Neurophysiol 102:1265-1273.

Herring BE, McMillan K, Pike CM, Marks J, Fox AP, Xie Z (2011) Etomidate and propofol inhibit the neurotransmitter release machinery at different sites. J Physiol 589:1103-1115.

Hoang B, Chiba A (2001) Single-cell analysis of Drosophila larval neuromuscular synapses. Dev Biol 229:55-70.

Kaeser PS, Regehr WG (2017) The readily releasable pool of synaptic vesicles. Curr Opin Neurobiol 43:63-70.

Karunanithi S, Barclay JW, Robertson RM, Brown IR, Atwood HL (1999) Neuroprotection at Drosophila synapses conferred by prior heat shock. J Neurosci 19:4360-4369.

Karunanithi S, Marin L, Wong K, Atwood HL (2002) Quantal size and variation determined by vesicle size in normal and mutant Drosophila glutamatergic synapses. J Neurosci 22:10267-10276.

Karunanithi S, Troup M, van Swinderen B (2018) Using Drosophila to understand general anesthesia: From synapses to behavior. Methods Enzymol 602:153-176.

Kim J, Yao A, Atherley R, Carstens E, Jinks SL, Antognini JF (2007) Neurons in the ventral spinal cord are more depressed by isoflurane, halothane, and propofol than are neurons in the dorsal spinal cord. Anesth Analg 105:1020-1026.

Kitamura A, Marszalec W, Yeh JZ, Narahashi T (2003) Effects of halothane and propofol on excitatory and inhibitory synaptic transmission in rat cortical neurons. J Pharmacol Exp Ther 304:162-171.

Kittel RJ, Wichmann C, Rasse TM, Fouquet W, Schmidt M, Schmid A, Wagh DA, Pawlu C, Kellner RR, Willig KI, Hell SW, Buchner E, Heckmann M, Sigrist SJ (2006) Bruchpilot promotes active zone assembly, $\mathrm{Ca} 2+$ channel clustering, and vesicle release. Science 312:1051-1054.

Korn H, Faber DS (1991) Quantal analysis and synaptic efficacy in the CNS. Trends Neurosci 14:439-445.

Koyanagi Y, Torturo CL, Cook DC, Zhou Z, Hemmings HC Jr (2019) Role of specific presynaptic calcium channel subtypes in isoflurane inhibition of synaptic vesicle exocytosis in rat hippocampal neurones. Br J Anaesth 123:219-227.

Kurdyak P, Atwood HL, Stewart BA, Wu CF (1994) Differential physiology and morphology of motor axons to ventral longitudinal muscles in larval Drosophila. J Comp Neurol 350:463-472.

Lavidis NA (1995) The effect of opiates on the secretion of transmitter from amphibian motor nerve terminals. Eur J Pharmacol 276:7176.

Lavidis NA, Bennett MR (1992) Probabilistic secretion of quanta from visualized sympathetic nerve varicosities in mouse vas deferens. J Physiol 454:9-26.

Leite LF, Gomez RS, Fonseca M. d. C, Gomez MV, Guatimosim C (2011) Effect of intravenous anesthetic propofol on synaptic vesicle exocytosis at the frog neuromuscular junction. Acta Pharmacol Sin 32:31-37.

Li X, Goel P, Wondolowski J, Paluch J, Dickman D (2018) A glutamate homeostat controls the presynaptic inhibition of neurotransmitter release. Cell Rep 23:1716-1727.

Lnenicka GA, Atwood HL, Marin L (1986) Morphological transformation of synaptic terminals of a phasic motoneuron by long-term tonic stimulation. J Neurosci 6:2252-2258.

McKinney SA, Murphy CS, Hazelwood KL, Davidson MW, Looger LL (2009) A bright and photostable photoconvertible fluorescent protein. Nat Methods 6:131-133.

McLachlan EM (1978) The statistics of transmitter release at chemical synapses. Int Rev Physiol 17:49-117.

Melom JE, Akbergenova Y, Gavornik JP, Littleton JT (2013) Spontaneous and evoked release are independently regulated at individual active zones. J Neurosci 33:17253-17263. 
Menon KP, Carrillo RA, Zinn K (2013) Development and plasticity of the Drosophila larval neuromuscular junction. Wiley Interdiscip Rev Dev Biol 2:647-670.

Metz LB, Dasgupta N, Liu C, Hunt SJ, Crowder CM (2007) An evolutionarily conserved presynaptic protein is required for isoflurane sensitivity in C. elegans. Anesthesiology 107:971-982.

Neher E, Brose N (2018) Dynamically primed synaptic vesicle states: Key to understand synaptic short-term plasticity. Neuron 100:12831291.

Newman ZL, Hoagland A, Aghi K, Worden K, Levy SL, Son JH, Lee LP, Isacoff EY (2017) Input-specific plasticity and homeostasis at the Drosophila larval neuromuscular junction. Neuron 93:13881404.e10.

Pawlu C, DiAntonio A, Heckmann M (2004) Postfusional control of quantal current shape. Neuron 42:607-618.

Regehr WG (2012) Short-term presynaptic plasticity. Cold Spring Harb Perspect Biol 4:a005702.

Schikorski T, Stevens CF (1997) Quantitative ultrastructural analysis of hippocampal excitatory synapses. J Neurosci 17:5858-5867.

Südhof TC, Rizo J (2011) Synaptic vesicle exocytosis. Cold Spring Harb Perspect Biol 3:a005637.

Ton HT, Phan TX, Abramyan AM, Shi L, Ahern GP (2017) Identification of a putative binding site critical for general anesthetic activation of TRPA1. Proc Natl Acad Sci U S A 114:37623767.

Troup M, Zalucki OH, Kottler BD, Karunanithi S, Anggono V, van Swinderen B (2019) Syntaxin1A neomorphic mutations promote rapid recovery from isoflurane anesthesia in Drosophila melanogaster. Anesthesiology 131:555-568.
Turrigiano GG, Leslie KR, Desai NS, Rutherford LC, Nelson SB (1998) Activity-dependent scaling of quantal amplitude in neocortical neurons. Nature 391:892-896.

van Swinderen B, Kottler B (2014) Explaining general anesthesia: A two-step hypothesis linking sleep circuits and the synaptic release machinery. Bioessays 36:372-381.

van Swinderen B, Saifee O, Shebester L, Roberson R, Nonet ML, Crowder CM (1999) A neomorphic syntaxin mutation blocks volatile-anesthetic action in Caenorhabditis elegans. Proc Natl Acad Sci U S A 96:2479-2484.

Wagh DA, Rasse TM, Asan E, Hofbauer A, Schwenkert I, Dürrbeck H, Buchner S, Dabauvalle M-C, Schmidt M, Qin G, Wichmann C, Kittel R, Sigrist SJ, Buchner E (2006) Bruchpilot, a protein with homology to ELKS/CAST, is required for structural integrity and function of synaptic active zones in Drosophila. Neuron 49:833-844.

Woll KA, Guzik-Lendrum S, Bensel BM, Bhanu NV, Dailey WP, Garcia BA, Gilbert SP, Eckenhoff RG (2018) An allosteric propofolbinding site in kinesin disrupts kinesin-mediated processive movement on microtubules. J Biol Chem 293:11283-11295.

Xie Z, McMillan K, Pike CM, Cahill AL, Herring BE, Wang Q, Fox AP (2013) Interaction of anesthetics with neurotransmitter release machinery proteins. J Neurophysiol 109:758-767.

Zalucki OH, Menon H, Kottler B, Faville R, Day R, Bademosi AT, Lavidis N, Karunanithi S, van Swinderen B (2015) Syntaxin1A-mediated resistance and hypersensitivity to isoflurane in Drosophila melanogaster. Anesthesiology 122:1060-1074.

Zhou C, Johnson KW, Herold KF, Hemmings HC Jr (2019) Differential inhibition of neuronal sodium channel subtypes by the general anesthetic isoflurane. J Pharmacol Exp Ther 369:200-211. 